\title{
Spontaneous Meckel's cave hematoma: A rare cause of trigeminal neuralgia
}

$\underline{\text { Concetta Alafaci, }}, \underline{\text { Giovanni Grasso, }}{ }^{1}$ Francesca Granata, ${ }^{2}$ Daniele Marino, Francesco M. Salpietro, and Francesco Tomasello

Department of Neurosurgery, University of Messina, Messina, Italy

${ }^{1}$ Department of Neurosurgery, University of Palermo, Palermo, Italy

${ }^{2}$ Department of Neuroradiology, University of Messina, Messina, Italy

Concetta Alafaci: calafaci@unime.it; Giovanni Grasso: giovanni.grasso@unipa.it; Francesca Granata: fgranata@unime.it; Daniele Marino:

marinodott.daniele@gmail.com; Francesco M. Salpietro: fsalp@unime.it; Francesco Tomasello: tomasello@unime.it

*Corresponding author

Received 2015 Jul 1; Accepted 2015 Jul 24.

Copyright : @ 2015 Surgical Neurology International

This is an open access article distributed under the terms of the Creative Commons Attribution-NonCommercial-ShareAlike 3.0 License, which allows others to remix, tweak, and build upon the work non-commercially, as long as the author is credited and the new creations are licensed under the identical terms.

\section{Abstract}

Go to:

\section{Background:}

The most common etiology of classic trigeminal neuralgia (TN) is vascular compression. However, other causes must be considered. Among these, spontaneous hematoma of the Meckel's cave (MC) causing symptomatic TN is very rare.

\section{Case Description:}

We present the case of a 60-year-old woman with a 2-month history of left TN and diplopia. Neuroradiological examinations revealed a well-defined hematoma in the left MC. The patient underwent surgical decompression with a progressive neurological improvement.

\section{Conclusion:}

Despite the number of lesions potentially affecting the MC, spontaneous hemorrhage is rare but should be taken into account in the differential diagnosis.

Keywords: Intracranial hemorrhage, Meckel's cave, trigeminal neuralgia

\section{INTRODUCTION}

Typical trigeminal neuralgia $(\mathrm{TN})$ is characterized by short periods of excruciating pain in a particular area of the face. The pain is paroxysmal and recurrent, lasting from a fraction of a second to few minutes. This occurrence has an estimated annual incidence of about 4.5/100,000 in the general population for both sexes.[10] The pain is usually triggered by a light touch to a specific area of the face or inside the mouth, and it does not respond to conventional analgesics. 
$\mathrm{TN}$ in its etiology is divided into a classic form, caused by vascular compression of the trigeminal nerve root, and symptomatic one, caused by other factors as tumors, vascular disorders, and demyelination in multiple sclerosis. $[\underline{5}, \underline{6}]$ To date, only two cases of TN related to spontaneous hematoma of the Meckel's cave (MC) have been described.[4, 12] Our report adds a new case with unique features and points out the importance of an early surgical treatment upon the diagnosis.

\section{Clinical presentation}

A 60-year-old woman was admitted with a 2-month history of the left mild TN and numbness on the left face. A double vision on the right lateral gaze was also reported. The neurological examination revealed a superficial hypoesthesia on the second branch of the left trigeminal nerve and a VI cranial nerve palsy. The patient's medical history was unremarkable except for slight systemic hypertension and unspecified surgery facelift occurred 2 years before admission. Magnetic resonance imaging (MRI) showed a round-shaped lesion, hyperintense in T1weighted sequences, occupying the left MC [igure 1]. The patient underwent computed tomography angiography scan, which was negative for any vascular malformations.

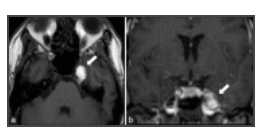

Figure 1

Preoperative axial (a) and coronal (b) nonenhanced T1-weighted magnetic resonance images showing a left high-signal Meckel's cave hematoma (arrow)

\section{Treatment}

The patient underwent surgical treatment. A left mini-pterional approach was performed, and a lumbar drainage was placed at the beginning of the procedure, to allow an optimal brain parenchyma relaxation. The dura of the MC was opened, and blood clots were removed achieving optimal decompression. Specimens were sent for histological examination.

The postoperative course was uneventful. The TN disappeared and the VI cranial nerve palsy gradually recovered. The sensory disturbances on the left part of the face improved, being scarcely observed at the 8-month follow-up. Histological examination did not show any pathological lesion but only erythrocytes and rare polymorphonuclear granulocytes, confirming the diagnosis of spontaneous hemorrhage.

Brain MRI, performed 8 months later, showed the complete disappearance of the hematoma [Figure 2].

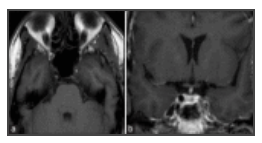

\section{Figure 2}

Postoperative axial (a) and coronal (b) T1-weighted magnetic resonance images confirming the complete removal of the hematoma

Although there is no proven etiology of TN, some autopsy results in patients with TN and multiple sclerosis indicate that demyelination within the descending tract of the trigeminal nerve or within the nerve itself can be correlated with the disorder.[9] The mechanism of the pain in nonmultiple sclerosis patients is still a matter of debate. Considerable surgical evidence, however, indicates that in many cases of $\mathrm{TN}$ the nerve is compressed by an artery or, less commonly, a vein in the posterior fossa, at the root entry/exit zone of the cranial nerve, a junctional area between central and peripheral myelin.[7] Over the past several years, this concept has been widely accepted and has stimulated several studies addressed primarily at establishing precise patients selection 
criteria for microvascular decompression in cranial nerve dysfunction. With the advent of MRI, which, using specific three-dimensional sequences, has offered a good visualization of both cranial nerves and cerebral vessels, neurovascular compression disorders have been diagnosed with increasing frequency, thus providing additional evidence supporting microvascular decompression treatment.

Besides vascular compression, other structural lesions such as tumors, $[\underline{1}, \underline{2}, \underline{3}]$ aneurysms or arteriovenous malformations, $[\underline{11}, \underline{14}]$ arachnoidal cyst $[\underline{8}]$ may cause TN. In our case, the TN was related to a spontaneous hematoma into the MC. This occurrence is very rare, being so far reported in only two cases.[4, $\underline{12}]$

The mechanisms for selective bleeding into the MC are unknown. It could be speculated that small tumors arising into the MC can bleed and destroy themselves by the acute hemorrhage so as histology cannot detect pathologic features. Beside this suggestive hypothesis, systemic hypertension should be considered as a possible cause. Nontraumatic intracerebral hemorrhage the most commonly results from hypertensive damage to blood vessel walls, but it also may be due to autoregulatory dysfunction with excessive cerebral blood flow. It is well known that chronic hypertension produces a small vessel vasculopathy. Such a vasculopathy is mainly characterized by lipohyalinosis, fibrinoid necrosis, and development of Charcot-Bouchard aneurysms, affecting penetrating arteries throughout the brain.[15] Predilection sites for intracerebral hemorrhage include the basal ganglia (40-50\%), lobar regions (20-50\%), thalamus (10-15\%), pons (5-12\%), cerebellum (5-10\%), and other brainstem sites (1-5\%).[15] Selective hemorrhage into the MC is very rare and considering the vascular anatomy of the $\mathrm{MC}$, a well-delimited bleeding may be related to small arterioles rupture surrounding the inner layer of dura located anteriorly, in relation to the cavernous sinus. This hypothesis could explain the bleeding inside the trigeminal cistern and the compression of the sixth cranial nerve as observed in our case. The blood clots removal reversed most of the symptoms observed before surgical decompression, including the sixth cranial nerve palsy.

Given the peculiarity of our findings, the reported case points out the importance of the knowledge of such a pathological condition when dealing with patients affected by TN. Accordingly, Meckel's cave hematoma should be considered, and a differential diagnosis should be made with hemorrhagic tumors such as trigeminal schwannomas or arteriovenous malformations in the MC.

MC pathology includes a large variety of tumors that are, in order of decreasing frequency, schwannomas, meningiomas, epidermoids, dermoids, hemangiopericytomas, metastases, lipomas, xanthomas, and lymphomas. The largest single-institution series so far reported by Muto et al. in 2010 includes 37 cases, most of them being schwannomas.[13] Despite this variety, lesions arising in the MC are rare and even more rare if of hemorrhagic nature.

\section{Financial support and sponsorship}

Nil.

\section{Conflicts of interest}

There are no conflicts of interest.

http://surgicalneurologyint.com/Spontaneous-Meckel's-cave-hematoma:-A-rare-cause-of-trigeminal-neuralgial

1. Alafaci C, Caffo M, Barresi V, Cutugno M, Pino MA, Granata F, et al. Large trigeminal schwannoma of the infratemporal fossa: Evaluation of neoangiogenesis in this rare neoplasm. Head Neck. 2013;35:E272-6.

[PubMed] 
2. Alafaci C, Salpietro FM, Puglisi E, Tripodo E, Matalone D, Di Pietro G, et al. Trigeminal pain caused by a cerebellopontine-angle lipoma. Case report and review of the literature. J Neurosurg Sci. 2001;45:110-3. [PubMed]

3. Cirak B, Kiymaz N, Arslanoglu A. Trigeminal neuralgia caused by intracranial epidermoid tumor: Report of a case and review of the different therapeutic modalities. Pain Physician. 2004;7:129-32. [PubMed]

4. Douvrin F, Ménif E, Callonnec F, Thiébot J. What is your diagnosis. Trigeminal neuralgia caused by a hematoma in Meckel's cavity? J Neuroradiol. 1999;26:213-4. [릴ed]

5. Evers S. The new IHS classification. Background and structure. Schmerz. 2004;18:351-6. [PubMed]

6. Farri A, Enrico A, Farri F. Headaches of otolaryngological interest: Current status while awaiting revision of classification. Practical considerations and expectations. Acta Otorhinolaryngol Ital. 2012;32:77-86.

[PMC free article] [PubMed]

7. Gardner WJ, Miklos MV. Response of trigeminal neuralgia to decompression of sensory root; discussion of cause of trigeminal neuralgia. J Am Med Assoc. 1959;170:1773-6. [PubMed]

8. Grasso G, Passalacqua M, Giambartino F, Cacciola F, Caruso G, Tomasello F. Typical trigeminal neuralgia by an atypical compression: Case report and review of the literature. Turk Neurosurg. 2014;24:82-5. [PubMed]

9. Jannetta PJ. Neurovascular compression in cranial nerve and systemic disease. Ann Surg. 1980;192:518-25. [PMC free article] [PubMed]

10. Katusic S, Williams DB, Beard CM, Bergstralh EJ, Kurland LT. Epidemiology and clinical features of idiopathic trigeminal neuralgia and glossopharyngeal neuralgia: Similarities and differences, Rochester, Minnesota, 1945-1984. Neuroepidemiology. 1991;10:276-81. [PubMed]

11. Levitt MR, Ramanathan D, Vaidya SS, Hallam DK, Ghodke BV. Endovascular palliation of AVM-associated intractable trigeminal neuralgia via embolization of the artery of the foramen rotundum. Pain Med.

2011;12:1824-30. [PubMed]

12. Maggioni F, Bellamio M, Margoni M, Zanchin G, Manara R. Hematoma of Meckel's cave: A rare possible symptomatic trigeminal neuralgia. J Neurol. 2012;259:1481-2. [PubMed]

13. Muto J, Kawase T, Yoshida K. Meckel's cave tumors: Relation to the meninges and minimally invasive approaches for surgery: Anatomic and clinical studies. Neurosurgery. 2010;67(3 Suppl Operative):291-8. [PubMed]

14. Wang C, Wang YN, Sun K, Yin J, Xiao HS, Zhong J. A successful treatment of coexistent trigeminal neuralgia and ipsilateral superior cerebellar artery aneurysm. J Craniofac Surg. 2015;26:1270-2. [PubMed]

15. Woo D, Haverbusch M, Sekar P, Kissela B, Khoury J, Schneider A, et al. Effect of untreated hypertension on hemorrhagic stroke. Stroke. 2004;35:1703-8. [PubMed] 